\title{
Comparative transcriptome analysis of miRNA in hydronephrosis male children caused by ureteropelvic junction obstruction with or without renal functional injury
}

\author{
Ge Liu ${ }^{1}$, Xin Liu ${ }^{1}$, Yi Yang ${ }^{\text {Corresp. } 1}$ \\ ${ }^{1}$ Urology Division, Pediatric Surgery Department, Shengjing Hospital of China Medical University, Shenyang, Liaoning, People's Republic of China \\ Corresponding Author: Yi Yang \\ Email address: yangy2@sj-hospital.org
}

MicroRNAs (miRNAs or miRs) are non-coding RNAs that contribute to pathological processes of various kidney diseases. Renal function injury represents a final common outcome of congenital obstructive nephropathy and has attracted a great deal of attention. However the molecular mechanisms are still not fully established. In this study, we compared transcriptome sequencing data of miRNAs of renal tissues from congenital hydronephrosis children with or without renal functional injury, in order to better understand whether microRNAs could play important roles in renal functional injury after ureteropelvic junction obstruction. A total of 22 microRNAs with significant changes in their expression were identified. 5 microRNAs were up-regulated and 17 microRNAs were downregulated in the renal tissues of the hydronephrosis patients with renal function injury compared with those without renal function injury. MicroRNA target genes were predicted by three major online miRNA target prediction algorithms, and all these mRNAs were used to perform the gene ontology analysis and Kyoto Encyclopedia of Gene and Genomes pathway analysis. Then twelve candidate human and rat homologous miRNAs were selected for validation using RT-qPCR in vitro and in vivo, only miR-187-3p had a trend identical to that detected by the sequencing results among the human tissues, in vivo and in vitro experimental models. In addition, we found that the change of miR-187-3p in vivo was consistent with results in vitro models and showed a decrease trend in time dependence. These results provided a detailed catalog of candidate miRNAs to investigate their regulatory role in renal injury of congenital hydronephrosis, indicating that they may serve as candidate biomarkers or therapeutic targets in the future. 
1 Comparative transcriptome analysis of miRNA in hydronephrosis male children caused by

2 ureteropelvic junction obstruction with or without renal functional injury

3

4

5

6

Ge Liu ${ }^{1}$, Xin Liu ${ }^{1}$, Yi Yang ${ }^{1}$

${ }^{1}$ Urology Division, Pediatric Surgery Department, Shengjing Hospital of China Medical University, Shenyang, Liaoning, China

Corresponding Author:

Yi Yang ${ }^{1}$

Urology Division, Pediatric Surgery Department, Shengjing Hospital of China Medical University, Shenyang, Liaoning, 110004, China

Email address: yangy2@sj-hospital.org

\section{Abstract}

MicroRNAs (miRNAs or miRs) are non-coding RNAs that contribute to pathological processes of various kidney diseases. Renal function injury represents a final common outcome of congenital obstructive nephropathy and has attracted a great deal of attention. However the molecular mechanisms are still not fully established. In this study, we compared transcriptome sequencing data of miRNAs of renal tissues from congenital hydronephrosis children with or without renal functional injury, in order to better understand whether microRNAs could play important roles in renal functional injury after ureteropelvic junction obstruction. A total of 22 microRNAs with significant changes in their expression were identified. 5 microRNAs were upregulated and 17 microRNAs were down-regulated in the renal tissues of the hydronephrosis patients with renal function injury compared with those without renal function injury.

MicroRNA target genes were predicted by three major online miRNA target prediction algorithms, and all these mRNAs were used to perform the gene ontology analysis and Kyoto Encyclopedia of Gene and Genomes pathway analysis. Then twelve candidate human and rat homologous miRNAs were selected for validation using RT-qPCR in vitro and in vivo, only miR-187-3p had a trend identical to that detected by the sequencing results among the human 
32 tissues, in vivo and in vitro experimental models. In addition, we found that the change of miR-

33 187-3p in vivo was consistent with results in vitro models and showed a decrease trend in time 34 dependence. These results provided a detailed catalog of candidate miRNAs to investigate their

35 regulatory role in renal injury of congenital hydronephrosis, indicating that they may serve as

36 candidate biomarkers or therapeutic targets in the future.

37

38

\section{Introduction}

Ureteropelvic junction obstruction (UPJO) is one of common clinical symptoms causing to persistent hydronephrosis in congenital hydronephrosis children. As a common pediatric urinary malformation, UPJO accounts for the majority of congenital urinary tract obstruction in the fetus, infants, and children(Kohaut and Tejani 1996). If there is no effective treatment, UPJO could cause to the progressive renal dysfunction(Jiang, Tang et al. 2017). UPJO is a congenital or acquired disease. Especially, congenital UPJO leads to chronic kidney disease (CKD) and endstage renal disease (ESRD) in children and infants (Chevalier 2004, Harambat, van Stralen et al. 2012), which represents a progressive irreversible structural damage and/or renal injury. Congenital UPJO affects millions of children's healthy in the world (Babu, Rathish et al. 2015). therefore, there is an urgent need to investigate the mechanisms of congenital UPJO onset and progression.

Over the last decade, many studies have been devoted to exploring the molecular mechanisms involved in the progression of CKD. However, the mechanisms of the development of renal fibrosis and functional damage are not clearly understood. MicroRNAs are a class of endogenous, small, non-coding RNAs that play an important role in regulating gene expression at the post-transcriptional level(Agrawal, Dasaradhi et al. 2003, Krol, Loedige et al. 2010). Previous clinical and experimental animal studies demonstrate that miRNAs play essential roles in the pathogenesis of various renal diseases (Oba, Kumano et al. 2010, Denby, Ramdas et al. 2014, Chung and Lan 2015, Loboda, Sobczak et al. 2016). However, these studies were either based on animal models or limited to a single miRNA of interest, and these studies did not compare the miRs in animal with that in human. 
62 In this study, we performed the transcriptome sequencing of miRNAs in kidney tissue, and 63 compared the data between normal differential renal function (DRF) and declined DRF infants 64 with ureteropelvic junction obstruction to better investigate the molecular mechanism of renal 65 injury in congenital hydronephrosis.

66

\section{Materials \& Methods}

\subsection{Patients and Samples}

From August 2016 to June 2017, 12 patients that are younger than 6 months with congenital unilateral hydronephrosis were selected as the research subjects. To confirm the diagnosis of hydronephrosis, all patients underwent urinary ultrasound and magnetic resonance urography (MRU), as well as Diuretic radionuclides (DRS) preoperatively. We divided the patients into two groups depending on their preoperative differential renal function (DRF), patients with normal DRF level $(>45 \%)$ were classified as normal DRF group while those with declined DRF level $(<30 \%)$ were considered as abnormal DRF group. All of them received open pyeloplasty, the ureteropelvic junctional obstruction were confirmed by operation and pathological postoperatively. The kidney parenchyma tissues were collected during surgery, according to the study of Song et al. (Song, Wang et al. 2019), and the tissues were frozen in liquid nitrogen and stored at $-80{ }^{\circ} \mathrm{C}$ for further study. The study was approved by the Ethics Committee of Shengjing Hospital, China Medical University, Shenyang, China (No. 2012PS81K), and the Ethics Committee waived the need for consent.

\subsection{Experimental Procedure of Unilateral Ureteral Obstruction}

The Unilateral Ureteral Obstruction (UUO) is a common prepared animal model in studying obstructive nephropathy. Two litters of 24 newborn Sprague Dawley (SD) rats (weight, 13-18 g, specific pathogen free) were obtained from Liaoning Changsheng Biotechnology Co. Ltd. (Benxi, China). Pups were kept with their dams in a laboratory animal facility with a constant temperature of $23 \pm 2{ }^{\circ} \mathrm{C}$ and relative humidity of $40 \pm 10 \%$ under a regular $12 \mathrm{~h} \mathrm{light/dark} \mathrm{cycle.}$ All animal care and experimental protocols were approved by the Committee on the Ethics of Animal Experiments of Shengjing Hospital, China Medical University, Shenyang, China (No. 2018PS228K).

One litter of pups were randomly divided into UUO-2d sham group and UUO-2d group ( $\mathrm{n}=6$, per group), the other litter of pups were randomly divided into UUO-5d sham group, and UUO- 
$935 \mathrm{~d}$ group ( $\mathrm{n}=6$, per group). Animal surgical procedures were performed using sterile technique 94 under isoflurane anaesthesia. The pups in the UUO groups underwent ligation of the left ureter 95 within $48 \mathrm{~h}$ after birth. Briefly, the left ureter was visualized by a flank incision, ligated with $8-0$ 96 silk below the renal pelvis and cut between the two ligatures. The sham group underwent the 97 same surgery except for left ureter ligation. Postoperatively, the pups were returned to their dams.

98 The criteria were established for euthanizing animals prior to the planned end of the experiment.

99 The pups were sacrificed with an overdose of pentobarbital sodium at $150 \mathrm{mg} / \mathrm{kg}$

100 intraperitoneally (i.p.) to reduce the pain or suffering; At days 2ed and 5th after surgery, the

101 kidneys were harvested, frozen in liquid nitrogen, and stored at $-80{ }^{\circ} \mathrm{C}$ until further processing.

$102 \quad 2.3$ Cell culture and Transform Growth Factor beta 1 (TGF- $\beta 1$ ) treatment

103 HK-2, a human kidney epithelial cell line, was purchased from the Shanghai Institute for

104 Biological Sciences (SIBS), Chinese Academy of Sciences (Shanghai, China) and cultured for

105 less than six months from the time of resuscitation. HK-2 cells were cultivated in Dulbecco's

106 modified Eagle's medium (DMEM) /F-12 1:1 (Hyclone Laboratories, Inc., Logan, UT)

107 supplemented $10 \%$ fetal bovine serum supplemented with $1 \%$ penicillin/streptomycin at $37^{\circ} \mathrm{C}$,

$1085 \% \mathrm{CO}_{2}$ in a humidified incubator. At $\sim 50 \%$ confluency, cells were treated with recombinant

109 human TGF- $\beta 1$ (10 ng/ml) (Proteintech Group, Inc) for 24 h, 48 h, 72 h, 96 h, 120 h and 144 h or

110 the vehicle control in DMEM/F12 1:1 for the indicated time period.

\section{$111 \quad 2.4$ MiRNA sequence}

112 Briefly, the kidney specimens were collected from hydronephrosis patients, and the RNA

113 sequencing libraries were constructed from the extracted and amplified RNA using the standard

114 Illumina library preparation protocols. miRNA-seq was performed on the Illumina HiSeq 2500

115 platform using PE150 protocol by Genesky Biotechnologies Inc (Shanghai, China). And data

116 were collected as previously described as Liu et al reported(Liu, Nie et al. 2017). And the tool of

117 ASprofle was performed for extraction and comparison of Alternative splicing (AS) events

118 (Florea, Song et al. 2013).

\section{$119 \quad 2.5$ Prediction of target genes}

120 The target genes of the differentially expressed miRNAs were screened by the starBase v2.0

121 (http://starbase.sysu.edu.cn) (Li, Liu et al. 2014), and gene searched in $>2$ databases

122 simultaneously, such as TargetScan (http://www.targetscan.org), miRTarBase 
123 (http://mirtarbase.mbc.nctu.edu.tw) or miRmap databases (http://mirmap.ezlab.org) were predicted

124 to be target genes.

\section{$125 \quad$ 2.6 Functional and pathway analysis}

126 For functional enrichment pathway analysis, gene ontology (GO) which was subdivided into

127 three groups, including biological processes, cellular components, and molecular functions, was

128 applied to analyze the function of the predicted target genes. Similarly, Kyoto Encyclopedia of

129 Genes and Genomes (KEGG) was used to find out the significant pathway of the predicted target

130 genes. The GO and KEGG analysis were performed using the OmicShare tools, a free online

131 platform for data analysis (http://www.omicshare.com/tools).

132 2.7 RNA Extraction and Validation of potential miRNAs using Real-Time Quantitative

133 Polymerase Chain Reaction (RT-qPCR).

134 Total RNA was extracted from kidney tissues of patients or rats and cultured cells by RNAiso

135 Plus (Takara, Shiga, Japan) according to the manufacturer's instructions. Total RNA (1 $\mu \mathrm{g})$ was

136 reverse-transcribed to cDNA by using the PrimeScript RT Reagent Kit (TaKaRa, Shiga, Japan)

137 following the manufacturer' s protocol. Then qRT-PCR was performed with the SYBR Premix

138 Ex Taq (TaKaRa, Shiga, Japan) and primers (Ribobio, Guangzhou, China) in a final volume of

$13920 \mu \mathrm{l}$. All PCR reactions were done in triplicates and repeated at least three times in a 7500 Real-

140 time PCR machine (Applied Biosystems, CA, USA). The relative miRNA levels were

141 normalized and quantified by U6 expression levels for each sample. Gene expression analysis

142 were performed using the $2^{-\Delta \Delta}$ Cycle thresholds $(\Delta \Delta \mathrm{Ct})$ method. All primers were designed and

143 synthesized by RiboBio Co., Ltd (Guangzhou, China).

\section{$144 \quad 2.8$ Statistical Analysis}

145 SPSS version 13.0 (SPSS Inc. Chicago, IL) and GraphPad Prism version 5.0 (GraphPad, San

146 Diego, Calif) were used to analyze the data, which are expressed as the mean \pm standard

147 deviation (SD). Differential expression of miRNAs in the kidney tissues of patients with UUO in

148 abnormal renal function group compared to those in normal renal function group was detected

149 using two-sided Student's t-test. P $<0.05$ was considered to be statistically significant.

150

151 Results

$152 \quad 3.1$ Clinical information 
153 All the patients with congenital hydronephrosis were Society of Fetal Urology (SFU) grade 4.

154 Among the 6 patients who underwent miRNA-seq, age distributions were 6-14 weeks in normal

155 renal function group (median age, 10 weeks) and 7-18 weeks in abnormal renal function group

156 (median age, 10 weeks). Meanwhile, the other 6 patients who underwent RT-qPCR validation

157 assays, age distributions were 12-20 weeks in normal renal function group (median age, 13

158 weeks) and 6-22 weeks in abnormal renal function group (median age, 12 weeks). All the

159 patients are male.

1603.2 Differentially expressed renal miRNAs in hydronephrosis children with or without 161 renal dysfunction

162 After comparative analysis of the miRNA-seq data, 22 miRNAs were differentially expressed in

163 kidney tissues of abnormal renal function group compared with normal renal function group

164 (fold change $>1.5 ; \mathrm{P}<0.01$; Table 1). Among them, 5 miRNAs were up regulation and 17

165 miRNAs were down regulation in hydronephrosis children with renal dysfunction, Hierarchical

166 clustered heatmap and Volcano plot showed the expression changes of miRNA between the two 167 groups (Figure 1).

\section{3.3 Construction of target prediction network of miRNA-mRNA}

169 To identify the function of the differentially expressed miRNAs, the prediction of miRNA target 170 genes was performed by the starBase v2.0 (http://starbase.sysu.edu.cn) (Li, Liu et al. 2014).

171 Subsequently, select genes that overlapped at least two of the three major online miRNA target

172 prediction algorithms: TargetScan, miRTarBase and miRmap databases. We performed the 22

173 differentially expressed miRNAs (Table 1) to predict the targeting genes using TargetScan,

174 miRTarBase and miRmap databases,1499 genes (Supplement 1) were obtained with overlapping

175 at least two of the three major online miRNA target prediction websides. All of these predicted

1761499 mRNAs were used to perform the GO and KEGG pathway analysis. As shown in Figure

$1772 \mathrm{~A}$, the most significant top $5 \mathrm{GO}$ terms in biological process were respectively cellular process,

178 biological regulation, metabolic process, regulation of biological process and response to

179 stimulus. In molecular function, the top $5 \mathrm{GO}$ terms were binding, catalytic activity, transcription

180 regular activity, molecular function regulator, and transporter activity. In cellular component,

181 cell, cell part, organelle, organelle part and membrane were the top 5 GO terms. A total of 64

182 distinct pathways with enrichment test $\mathrm{P}<0.05$ were identified according to the KEGG pathway

183 database. In addition, KEGG pathway analysis revealed that the identified miRNAs regulated the 
184 signaling pathways of cancer, Cushing Syndrome, Longevity regulation, regulation of

185 pluripotency of stem cells, axon guidance, etc. (Figures 2B).

$186 \quad 3.4$ Validation of the microarray data through RT-qPCR in neonatal rat kidney tissues 187 with UUO

188 To validate the microarray data, twelve candidate human and rat homologous miRNAs were selected for miRNA expression validation using RT-qPCR, including four up-regulated miRNAs

190 (miR-21-5p, miR-224-5p, miR-142-5p, miR-212-5p) and eight down-regulated miRNAs (miR-

191 378a-5p, miR-138-5p, miR-874-3p and miR-378a-3p miR-187-3p, miR-204-3p, miR-873-3p,

192 miR-338-3p) (Table 2). In neonatal rat kidney tissues with 2-day UUO, we found that miR-187-

$1933 p$, miR-873-3p, and miR-21-5p in twelve selected miRNAs were all up-regulated with statistical

194 significance. Only the trend of change in miR-21-5p was consistent with that observed by

195 miRNA-Seq data (Figure 3A). Furthermore, in neonatal rat kidney tissues with 5-day UUO, we

196 found that the change trends of one (miR-187-3p) from the down-regulated miRNAs and two

197 from the up-regulated ones (miR-21-5p, miR-142-5p) revealed the same expression tendency as

198 miRNA-Seq data and were statistically significant (Figure 3B). Therefore, miR-21-5p, miR-187-

$1993 \mathrm{p}, \mathrm{miR}-142-5 \mathrm{p}$ were selected for further validation in kidney tissues of patients with congenital

200 hydronephrosis.

$201 \quad 3.5$ Validation of the microarray data through RT-qPCR in kidney tissues of patients with 202 congenital hydronephrosis

203 We further analyzed the miRNA expression levels of miR-187-3p, miR-21-5p and miR-142-5p

204 in kidney tissues of patients with congenital hydronephrosis using RT-qPCR. The expression

205 levels of miR-187-3p was significantly down-regulated in patients with abnormal DRF compared 206 to normal DRF ones (fold change $=0.277, \mathrm{p}=0.044$ ). Meanwhile, the expression level of miR-21$2075 \mathrm{p}$ was up-regulated but it was not statistically significant (Figure 3C). Moreover, there is no 208 significant difference in miR-142-5p expression between abnormal DRF group and normal DRF 209 group (Figure 3C).

210 3.6 Validation of the microarray data through RT-qPCR in HK-2 cells induced by TGF- $\beta 1$

211 in vitro

212 RT-qPCR analysis revealed that miR-187-3p expression was significantly up-regulated in HK-2

213 cells after induced by TGF- $\beta 1$ in vitro, and the expression level of miR-187-3p decreased with

214 time of induction (Figure 3D). As shown in Fig. 3D, the expression levels of miR-187-3p in HK- 
2152 cells induced by TGF- $\beta 1$ in vitro were obviously higher than those of control group with $216 \sim 10.68$-fold, $\sim 6.49$-fold, $\sim 4.20$-fold at 24, 48, $72 \mathrm{~h}$, respectively. However, at 96 and $120 \mathrm{~h}$ under

217 TGF- $\beta 1$ treatment, the expression level of miR-187-3p showed no significant difference between 218 the two groups $(p>0.05$ ). Induced for $144 \mathrm{~h}$, there was a marked decrease in expression of miR$219 \quad 187-3 p($ fold change $=0.001 ; p=0.027)$.

220

221

222

223

224

225

226

227

228

229

230

231

232

233

234

235

236

237

238

239

240

241

242

243

244

245

\section{Discussion}

Congenital obstructive nephropathy is a principal cause of renal failure in the pediatric population (Collins, Foley et al. 2008). The pathophysiological mechanism of obstruction nephropathy is very complex and diverse, involving cell apoptosis, oxidative stress, inflammation, and which altogether lead to progressive renal interstitial fibrosis (RIF). The development of RIF is linked to progressive renal injury and CKD (Falke, Gholizadeh et al. 2015). The permanent renal injury, even the obstruction is released by surgical corrections, cannot be prevented in some cases. To date, there are no effective treatments to reverse renal injury after obstruction. Therefore, a better understanding of the pathophysiological mechanism of renal function injury after UUO is required for developing more effective therapeutic strategies.

miRNAs are small non-coding RNA molecules that negatively regulate their target genes at the post-transcriptional level (Trionfini, Benigni et al. 2015). During the last decade, a growing number of studies have been undertaken to investigate miRNAs functions (Trionfini, Benigni et al. 2015). They are involved in numerous physiological and pathological processes, such as organ development, cancer progression and inflammatory diseases. There are many evidences that miRNAs play a crucial part in a variety of kidney diseases, such as renal cancer, $\operatorname{IgA}$ nephropathy, acute kidney injury and diabetes nephropathy (Gottardo, Liu et al. 2007, Kolfschoten, Roggli et al. 2009) (Hennino, Buob et al. 2016, Qin, Wang et al. 2019) In humans and animal disease models, altered miRNA expression has been demonstrated in fibrotic disorders of the lung, liver, and kidney (Vettori, Gay et al. 2012). However, their role in congenital obstructive nephropathy is still in infancy. In order to better understand the mechanism of renal function injury in obstructive nephropathy, we identified the miRNA 
246 transcriptome of kidney tissues of congenital hydronephrosis infants with or without renal

247 function injury for the first time in the present study.

248

249 Age and complementary food have been shown to influence miRNA expression(Elhassan,

250 Christie et al. 2012, Meder, Backes et al. 2014, Ameling, Kacprowski et al. 2015). In order to

251 avoid the influence of different complementary food on the expression of miRNA in kidney, we

252 selected patients younger than 6 months old with hydronephrosis as the research subjects. A total

253 of 22 differentially expressed miRNAs were detected in kidney tissues between the normal and

254 abnormal renal function groups.

255

256 The investigations of the miRNA-related functions require further validation in animal models, 257 and miRNA expression profiles may differ in various tissues and species (Zhou, Quan et al.

258 2018). Therefore, we selected 12 homologous miRNAs of human and rat for further validation in

259 preparation of kidneys of UUO rat models to confirm the accuracy of miRNA sequencing 260 results.

261 Because it is difficult to obtain human kidney specimens of obstructive nephropathy, unless the

262 patients have progressed to end-stage renal disease and underwent nephrectomy, the pathological

263 changes within the kidney to the process of renal functional injury cannot be easily addressed.

264 Hence, a variety of experimental models have been developed to study the pathophysiological

265 mechanism in obstructive nephropathy. The UUO model is widely used to study because the

266 procedure has similar characteristics to human obstructive nephropathy(Martínez-Klimova,

267 Aparicio-Trejo et al. 2019). There is inter-individual difference about the obstruction of ureters,

268 therefore, we chose complete unilateral ureteral obstruction in the rat model to induce renal

269 injury. Previously researches have shown that nephrogenesis proceeds postnatally in the neonatal

270 rats or mice (Hartman, Lai et al. 2007). This view was supported by Chevalier RL et al. in 2009,

271 who wrote that based on the duration of nephrogenesis, the rat or mouse at birth parallels the

272 midtrimester human, and the 7-day-old rat or mouse is analogous to the full-term human at birth

273 (Chevalier, Forbes et al. 2009). Therefore, the neonatal rat UUO model was chosen for this

274 study. Besides, Chevalier RL's data indicated that relief of the obstruction after two to five days

275 permitted recovery of renal structure and function (Chevalier, Thornhill et al. 2002). Therefore,

276 we collected the rat kidney samples on two and five days after UUO, which might be related to 
277 renal function injury phase. We performed qRT-PCR to confirm the differential expression of the 278 miRNAs between sham group and UUO groups. The results showed that the tendencies of miR279 874-3p, miR-204-3p, and miR-212-5p aligned with the results in previous miRNA sequence, but 280 these changes did not reach statistical significance. miR-187-3p was down-regulated and miR$28121-5 p$ and miR-142-5p were up-regulated, which agreed with the miRNA sequence results. 282 Interestingly, the expression of miR-187-3p was up-regulated in rat kidney tissues on 2 days 283 after UUO and down-regulated on 5 days after UUO.

It has been demonstrated that miR-21 including miR-21-5p plays an important role in renal fibrosis ( $\mathrm{Li}$, Wang et al. 2020). Besides, in the study by Ma Z, et al., miR-142-5p targets Smad3 thereby suppressing TGF-3-induced growth inhibition in cancer cells (Ma, Liu et al. 2016). The validation results in kidney tissues of patients showed that although the expression of miR-21-5p and miR-142-5p differed between sham rat and UUO rat, no differences were found in the kidney tissues of infants with congenital hydronephrosis. This could be related to the design of our study, the complete UUO model was chosen for this study, which could lead to reduced renal blood flow, interstitial fibrosis and reduced glomerular filtration rate (Vaughan, Marion et al. 2004). However, the ureteral obstruction is usually not complete obstruction in children with hydronephrosis, the severity of obstruction and the degree of injury are variable, the number of patients was small and there might have been selection bias. These may be the possible reason for this result. Transforming growth factor- $\beta$ is the strongest known fiber genic factor and plays an important role in the pathogenesis of renal interstitial fibrosis (Feger, Alesutan et al. 2015). Treatment of renal epithelial cells with TGF- $\beta 1$ is a model commonly used to study renal interstitial fibrosis (Liu 2004). TGF- $\beta$ is also a key EMT inducer that up-regulates important transcription factors in EMT. In this study, we used TGF- $\beta 1$ to establish a cell fibrosis model in vitro. It was worth noting that up-regulation of miR-187-3p in early stage (24-72 h) after TGF- $\beta$ stimulation has been observed in HK-2 cells, then gradually decreased, besides, the expression of miR-187-3p was down-regulated on day $6^{\text {th }}$ after TGF- $\beta$ stimulation. We speculate that the expression of miR-187-3p was initiated by UUO, it could play a positive role in protecting renal function, with the progression of the disease, miR-187-3p was consumed gradually till downregulated by $144 \mathrm{~h}$, indicating that miR-187-3p may serve a role in the development of renal injury. And its expression could be a biomarker of renal function injury in infants with 
308 congenital hydronephrosis, However, this hypothesis needs to be further verified using more in 309 vivo and in vitro experiments.

310

311 Following that, we analyzed the predicted 1499 genes from 22 differentially expressed miRNA

312 by GO and KEGG. We demonstrated that some important predicted genes were repeated in top

31320 pathways, including PIK3R1, MAPK1, IGF1, IGF1R and AKT3. And their related signaling

314 pathways including FoxO signaling pathway, PI3K-Akt signaling pathway and Hippo signaling

315 pathway, have been reported by other researchers to regulate the renal inflammation and fibrosis

316 in CKD (Yoon, Kim et al. 2014, Patel, Tang et al. 2019). Among those differentially expressed

317 miRNAs, miR-187-3p was reported to participate in regulating the many different biological

318 processes, especially in cancer progression (Dou, Liu et al. 2016)(Sun, Li et al. 2016, Zhang, Luo

319 et al. 2016). Moreover, Zhang F, et al. demonstrated that miR-187 inhibited the epithelial-

320 mesenchymal transition (EMT) process via repressing the TGF- $\beta /$ Smad pathway through

321 targeting NT5E and PTK6 in colorectal carcinoma (Zhang, Luo et al. 2016); Dou et al also

322 demonstrated that miR-187-3p could suppressed the metastasis and EMT of hepatocellular

323 carcinoma (Dou, Liu et al. 2016). Although the mechanism by which congenital obstructive

324 nephropathy produced renal injury was not completely understood, it has been suggested that

325 TGF- $\beta 1$-induced epithelial-to-mesenchymal transition plays a key role in renal injury in

326 obstructive nephropathy (Chevalier, Thornhill et al. 2010). And epithelial-to-mesenchymal

327 transition was reported to be an important phenotype during CKD. EMT in tubule is a key

328 hallmark in obstructed kidneys. Tubular cells dedifferentiate to fibroblasts and move to the

329 interstitium leading to obstructed kidneys(Yang and Liu 2001, Iwano, Plieth et al. 2002). In the

330 present study, the expression of miR-187-3p was up-regulated in rat kidney tissues after UUO

331 and HK-2 cells after TGF- $\beta$ stimulation at the early time points, however, miR-187-3p was

332 down-regulated at the late time points. Consistently, we observed that miR-187-3p was down-

333 regulated in renal tissues of congenital hydronephrosis patients with renal function injury.

334 Therefore, miR-187-3p may perform the inhibitory role of EMT to regulate processes of renal

335 injury following ureteral obstruction, and it may be a novel therapeutic target for UUO induced

336 renal injury. However, there is still more validation experiments to investigate the mechanism of

337 miR-187-3p on UJPO.

338 


\section{Conclusions}

340 In this study, we have performed a miRNAs transcriptional analysis of kidney tissues of

341 congenital hydronephrosis infants with or without renal function injury, in order to track changes

342 in miRNAs expression that may be associated with renal injury in obstructive nephropathy.

343 These miRNAs could potentially serve as novel biological markers for indicating damaged renal

344 function. In the future, the role of these miRNAs in processes such as apoptosis, proliferation,

345 and differentiation should be determined, as well as its involvement in congenital obstructive

346 nephropathy, and we shall explore the in-depth mechanism to provide additional targets for

347 therapeutic intervention.

348

349 Acknowledgements

350 The authors will thank Fujiang Ma and Xueyan Li for their great help in RNA collection and RT351 qPCR analysis.

352

353 Data availability

354 The authors declare that all data are available in the article file. Source data are provided with 355 this paper.

356

357 Conflicts of Interest

358 The authors declare that they have no competing interests.

359

360

\section{Source of Funding}

361 This work was supported by National Natural Science Foundation of China, No. 81571514 and

362 Key Research and Development Program of Liaoning Province of China, No. 2020JH

$3632 / 10300145$.

364

365

366

\section{References} 657-685. 
371 Ameling, S., T. Kacprowski, R. K. Chilukoti, C. Malsch, V. Liebscher, K. Suhre, M. Pietzner, N.

372 Friedrich, G. Homuth, E. Hammer and U. Völker (2015). "Associations of circulating plasma

373

374

375

376

377

378

379

380

381

382

383

384

385

386

387 microRNAs with age, body mass index and sex in a population-based study." BMC Med Genomics 8: 61.

Babu, R., V. R. Rathish and V. Sai (2015). "Functional outcomes of early versus delayed pyeloplasty in prenatally diagnosed pelvi-ureteric junction obstruction." J Pediatr Urol 11(2):

63.e61-65.

Chevalier, R. L. (2004). "Biomarkers of congenital obstructive nephropathy: past, present and future." J Urol 172(3): 852-857.

Chevalier, R. L., M. S. Forbes and B. A. Thornhill (2009). "Ureteral obstruction as a model of renal interstitial fibrosis and obstructive nephropathy." Kidney Int 75(11): 1145-1152.

Chevalier, R. L., B. A. Thornhill, A. Y. Chang, F. Cachat and A. Lackey (2002). "Recovery from release of ureteral obstruction in the rat: relationship to nephrogenesis." Kidney Int 61(6): 20332043.

Chevalier, R. L., B. A. Thornhill, M. S. Forbes and S. C. Kiley (2010). "Mechanisms of renal injury and progression of renal disease in congenital obstructive nephropathy." Pediatr Nephrol 25(4): 687-697.

388 Chung, A. C. and H. Y. Lan (2015). "MicroRNAs in renal fibrosis." Front Physiol 6: 50.

389 Collins, A. J., R. Foley, C. Herzog, B. Chavers, D. Gilbertson, A. Ishani, B. Kasiske, J. Liu, L. W. 390 Mau, M. McBean, A. Murray, W. St Peter, J. Xue, Q. Fan, H. Guo, Q. Li, S. Li, S. Li, Y. Peng, Y. 391 Qiu, T. Roberts, M. Skeans, J. Snyder, C. Solid, C. Wang, E. Weinhandl, D. Zaun, R. Zhang, C. 392 Arko, S. C. Chen, F. Dalleska, F. Daniels, S. Dunning, J. Ebben, E. Frazier, C. Hanzlik, R.

393 Johnson, D. Sheets, X. Wang, B. Forrest, E. Constantini, S. Everson, P. Eggers and L. Agodoa (2008). "Excerpts from the United States Renal Data System 2007 annual data report." Am J Kidney Dis 51(1 Suppl 1): S1-320.

Denby, L., V. Ramdas, R. Lu, B. R. Conway, J. S. Grant, B. Dickinson, A. B. Aurora, J. D. McClure, D. Kipgen, C. Delles, E. van Rooij and A. H. Baker (2014). "MicroRNA-214 antagonism protects against renal fibrosis." J Am Soc Nephrol 25(1): 65-80. Dou, C., Z. Liu, M. Xu, Y. Jia, Y. Wang, Q. Li, W. Yang, X. Zheng, K. Tu and Q. Liu (2016). "miR-187-3p inhibits the metastasis and epithelial-mesenchymal transition of hepatocellular carcinoma by targeting S100A4." Cancer Lett 381(2): 380-390.

Elhassan, M. O., J. Christie and M. S. Duxbury (2012). "Homo sapiens systemic RNA interference-defective-1 transmembrane family member 1 (SIDT1) protein mediates contactdependent small RNA transfer and microRNA-21-driven chemoresistance." J Biol Chem 287(8): 5267-5277.

Falke, L. L., S. Gholizadeh, R. Goldschmeding, R. J. Kok and T. Q. Nguyen (2015). "Diverse origins of the myofibroblast-implications for kidney fibrosis." Nat Rev Nephrol 11(4): 233-244. Feger, M., I. Alesutan, T. Castor, S. Mia, K. Musculus, J. Voelkl and F. Lang (2015). "Inhibitory effect of $\mathrm{NH} 4 \mathrm{Cl}$ treatment on renal Tgfß1 signaling following unilateral ureteral obstruction." Cell Physiol Biochem 37(3): 955-964.

Florea, L., L. Song and S. L. Salzberg (2013). "Thousands of exon skipping events differentiate among splicing patterns in sixteen human tissues." F1000Res 2: 188.

Gottardo, F., C. G. Liu, M. Ferracin, G. A. Calin, M. Fassan, P. Bassi, C. Sevignani, D. Byrne, M. Negrini, F. Pagano, L. G. Gomella, C. M. Croce and R. Baffa (2007). "Micro-RNA profiling in kidney and bladder cancers." Urol Oncol 25(5): 387-392.

Harambat, J., K. J. van Stralen, J. J. Kim and E. J. Tizard (2012). "Epidemiology of chronic kidney disease in children." Pediatr Nephrol 27(3): 363-373.

Hartman, H. A., H. L. Lai and L. T. Patterson (2007). "Cessation of renal morphogenesis in

419 mice." Dev Biol 310(2): 379-387. 
420 Hennino, M. F., D. Buob, C. Van der Hauwaert, V. Gnemmi, Z. Jomaa, N. Pottier, G. Savary, E.

421

422

423

424

425

426

427

428

429

430

431

432

433

434

435

436

437

438

439

440

441

442

443

444

445

446

447

448

449

450

451

452

453

454

455

456

457

458

459

460

461

462

463

464

465

466

467

468

469

Drumez, C. Noël, C. Cauffiez and F. Glowacki (2016). "miR-21-5p renal expression is associated with fibrosis and renal survival in patients with IgA nephropathy." Sci Rep 6: 27209. Iwano, M., D. Plieth, T. M. Danoff, C. Xue, H. Okada and E. G. Neilson (2002). "Evidence that fibroblasts derive from epithelium during tissue fibrosis." The Journal of clinical investigation 110(3): 341-350.

Jiang, D., B. Tang, M. Xu, H. Lin, L. Jin, L. He, G. Xu, X. Fang and H. Geng (2017). "Functional and Morphological Outcomes of Pyeloplasty at Different Ages in Prenatally Diagnosed Society of Fetal Urology Grades 3-4 Ureteropelvic Junction Obstruction: Is It Safe to Wait?" Urology 101: 45-49.

Kohaut, E. C. and A. Tejani (1996). "The 1994 annual report of the North American Pediatric Renal Transplant Cooperative Study." Pediatr Nephrol 10(4): 422-434.

Kolfschoten, I. G., E. Roggli, V. Nesca and R. Regazzi (2009). "Role and therapeutic potential of microRNAs in diabetes." Diabetes Obes Metab 11 Suppl 4: 118-129.

Krol, J., I. Loedige and W. Filipowicz (2010). "The widespread regulation of microRNA biogenesis, function and decay." Nat Rev Genet 11(9): 597-610.

Li, J. H., S. Liu, H. Zhou, L. H. Qu and J. H. Yang (2014). "starBase v2.0: decoding miRNAceRNA, miRNA-ncRNA and protein-RNA interaction networks from large-scale CLIP-Seq data." Nucleic Acids Res 42(Database issue): D92-97.

Li, N., Z. Wang, F. Gao, Y. Lei and Z. Li (2020). "Melatonin ameliorates renal fibroblastmyofibroblast transdifferentiation and renal fibrosis through miR-21-5p regulation." $\mathrm{J}$ Cell Mol Med 24(10): 5615-5628.

Liu, Y. (2004). "Epithelial to mesenchymal transition in renal fibrogenesis: pathologic significance, molecular mechanism, and therapeutic intervention." J Am Soc Nephrol 15(1): 1-12. Liu, Y., H. Nie, C. Liu, X. Zhai, Q. Sang, Y. Wang, D. Shi, L. Wang and Z. Xu (2017). "Angulin proteins ILDR1 and ILDR2 regulate alternative pre-mRNA splicing through binding to splicing factors TRA2A, TRA2B, or SRSF1." Sci Rep 7(1): 7466.

Loboda, A., M. Sobczak, A. Jozkowicz and J. Dulak (2016). "TGF-beta1/Smads and miR-21 in Renal Fibrosis and Inflammation." Mediators Inflamm 2016: 8319283.

Ma, Z., T. Liu, W. Huang, H. Liu, H. M. Zhang, Q. Li, Z. Chen and A. Y. Guo (2016). "MicroRNA regulatory pathway analysis identifies miR-142-5p as a negative regulator of TGF- $\beta$ pathway via targeting SMAD3." Oncotarget 7(44): 71504-71513.

Martínez-Klimova, E., O. E. Aparicio-Trejo, E. Tapia and J. Pedraza-Chaverri (2019). "Unilateral Ureteral Obstruction as a Model to Investigate Fibrosis-Attenuating Treatments." Biomolecules 9(4).

Meder, B., C. Backes, J. Haas, P. Leidinger, C. Stähler, T. Großmann, B. Vogel, K. Frese, E. Giannitsis, H. A. Katus, E. Meese and A. Keller (2014). "Influence of the confounding factors age and sex on microRNA profiles from peripheral blood." Clin Chem 60(9): 1200-1208.

Oba, S., S. Kumano, E. Suzuki, H. Nishimatsu, M. Takahashi, H. Takamori, M. Kasuya, Y. Ogawa, K. Sato, K. Kimura, Y. Homma, Y. Hirata and T. Fujita (2010). "miR-200b precursor can ameliorate renal tubulointerstitial fibrosis." PLoS One 5(10): e13614.

Patel, S., J. Tang, J. M. Overstreet, S. Anorga, F. Lian, A. Arnouk, R. Goldschmeding, P. J. Higgins and R. Samarakoon (2019). "Rac-GTPase promotes fibrotic TGF- $\beta 1$ signaling and chronic kidney disease via EGFR, p53, and Hippo/YAP/TAZ pathways." Faseb j 33(9): 97979810.

Qin, Y., G. Wang and Z. Peng (2019). "MicroRNA-191-5p diminished sepsis-induced acute kidney injury through targeting oxidative stress responsive 1 in rat models." Biosci Rep 39(8).

Song, J., T. Wang, X. Chi, X. Wei, S. Xu, M. Yu, H. He, J. Ma, X. Li and J. Du (2019). "Kindlin-2 inhibits the hippo signaling pathway by promoting degradation of MOB1." Cell reports 29(11): 3664-3677. e3665.

PeerJ reviewing PDF | (2021:01:57453:1:2:NEW 4 Jan 2022) 
470 Sun, C., S. Li, C. Yang, Y. Xi, L. Wang, F. Zhang and D. Li (2016). "MicroRNA-187-3p mitigates non-small cell lung cancer (NSCLC) development through down-regulation of BCL6." Biochem Biophys Res Commun 471(1): 82-88.

473 Trionfini, P., A. Benigni and G. Remuzzi (2015). "MicroRNAs in kidney physiology and disease." $474 \quad$ Nat Rev Nephrol 11(1): 23-33.

475 Vaughan, E. D., Jr., D. Marion, D. P. Poppas and D. Felsen (2004). "Pathophysiology of 476 unilateral ureteral obstruction: studies from Charlottesville to New York." J Urol 172(6 Pt 2):

477 2563-2569.

478 Vettori, S., S. Gay and O. Distler (2012). "Role of MicroRNAs in Fibrosis." Open Rheumatol J 6: 479 130-139.

480 Yang, J. and Y. Liu (2001). "Dissection of key events in tubular epithelial to myofibroblast 481 transition and its implications in renal interstitial fibrosis." The American journal of pathology 482 159(4): 1465-1475.

483 Yoon, H. E., S. J. Kim, S. J. Kim, S. Chung and S. J. Shin (2014). "Tempol attenuates renal 484 fibrosis in mice with unilateral ureteral obstruction: the role of PI3K-Akt-FoxO3a signaling." $\underline{\mathrm{J}}$ 485 Korean Med Sci 29(2): 230-237.

486 Zhang, F., Y. Luo, Z. Shao, L. Xu, X. Liu, Y. Niu, J. Shi, X. Sun, Y. Liu, Y. Ding and L. Zhao 487 (2016). "MicroRNA-187, a downstream effector of TGF $\beta$ pathway, suppresses Smad-mediated 488 epithelial-mesenchymal transition in colorectal cancer." Cancer Lett 373(2): 203-213.

489 Zhou, L., S. Quan, H. Xu, L. Ma and J. Niu (2018). "Identification and Expression of miRNAs Related to Female Flower Induction in Walnut (Juglans regia L.)." Molecules 23(5). 


\section{Table $\mathbf{1}$ (on next page)}

Fold change expression of 22 differentially expressed miRNAs in renal tissues of congenital hydronephrosis infants from RNA-seq data.

Fold change expression of 22 differentially expressed miRNAs( fold change $>1.5 ; \mathrm{P}<0.01$ ) in renal tissues of congenital hydronephrosis infants from RNA-seq data. 
1 Table 1 Fold change expression of 22 differentially expressed miRNAs in

2 renal tissues of congenital hydronephrosis infants from RNA-seq data.

\begin{tabular}{|c|c|c|c|c|}
\hline miRNA & DNF $>45 \%$ & DNF $<35 \%$ & pvalue & log2FoldChange \\
\hline hsa-miR-7704 & $18.35 \pm 16.43$ & $2.46 \pm 2.39$ & 0.003613634 & -2.974923849 \\
\hline hsa-miR-3195 & $33.83 \pm 28.28$ & $5.03 \pm 2.07$ & 0.00091878 & -2.753388116 \\
\hline hsa-miR-378a-5p & $226.17 \pm 151.78$ & $43.13 \pm 20.66$ & 0.000191141 & -2.377348869 \\
\hline hsa-miR-138-5p & $243.66 \pm 165.81$ & $47.64 \pm 32.7$ & 0.001229062 & -2.341418947 \\
\hline hsa-miR-378d & $1232.59 \pm 906.44$ & $243.52 \pm 91.08$ & 0.000365338 & -2.337746685 \\
\hline hsa-miR-7641 & $631.08 \pm 742.43$ & $130.48 \pm 28.85$ & 0.002161003 & -2.269745727 \\
\hline hsa-miR-3656 & $193.71 \pm 183.34$ & $43.37 \pm 17$ & 0.001779968 & -2.153430132 \\
\hline hsa-miR-378c & $2191.15 \pm 1535.8$ & $524.84 \pm 167.88$ & 0.000907617 & -2.060900181 \\
\hline hsa-miR-378i & $154.42 \pm 84.7$ & $40.58 \pm 22.45$ & 0.003083273 & -1.920454026 \\
\hline hsa-miR-378f & $51.55 \pm 29.27$ & $13.67 \pm 4.74$ & 0.004442206 & -1.884860173 \\
\hline hsa-miR-204-3p & $128.99 \pm 86.44$ & $37.53 \pm 12.74$ & 0.006674413 & -1.768711872 \\
\hline hsa-miR-187-3p & $375.69 \pm 245.62$ & $111.26 \pm 45.93$ & 0.006219278 & -1.752649274 \\
\hline hsa-miR-873-3p & $90.82 \pm 57.77$ & $26.6 \pm 12.54$ & 0.008710633 & -1.750311834 \\
\hline hsa-miR-378a-3p & $11471.13 \pm 6230.49$ & $3543.99 \pm 1894.21$ & 0.004923984 & -1.694418411 \\
\hline hsa-miR-874-3p & $21027.61 \pm 10491.11$ & $6795.22 \pm 2486.92$ & 0.002100211 & -1.629586819 \\
\hline hsa-miR-338-3p & $342.78 \pm 215.06$ & $113.12 \pm 38.23$ & 0.009335378 & -1.594507199 \\
\hline hsa-miR-4532 & $1383.65 \pm 830.96$ & $465.35 \pm 202.48$ & 0.006808167 & -1.572280994 \\
\hline hsa-miR-212-5p & $66.42 \pm 53.3$ & $220.92 \pm 108.49$ & 0.007819275 & 1.718296369 \\
\hline hsa-miR-224-5p & $59.88 \pm 53$ & $235.67 \pm 71.48$ & 0.002229173 & 1.955015383 \\
\hline hsa-miR-142-5p & $263.33 \pm 188.08$ & $1076.71 \pm 772.04$ & 0.002629789 & 2.027340006 \\
\hline hsa-miR-31-5p & $53.08 \pm 56.68$ & $326.16 \pm 350.43$ & 0.002166778 & 2.606070929 \\
\hline hsa-miR-21-5p & $27210.04 \pm 18167.04$ & $181317.86 \pm 212736.06$ & 0.001015935 & 2.736284032 \\
\hline
\end{tabular}




\section{Table 2 (on next page)}

human and rat homologous miRNAs sequence

* represents up-regulated miRNA in miRNA-Seq data 
1 Table 2. human and rat homologous miRNAs sequence

\begin{tabular}{ccc}
\hline miRNA & Human & Rat \\
\hline miR-378a-5p & CUCCUGACUCCAGGUCCUGUGU & CUCCUGACUCCAGGUCCUGUGU \\
miR-138-5p & AGCUGGUGUUGUGAACAGGCCG & AGCUGGUGUUGUGAAUCAGGCCG \\
miR-874-3p & CUGCCCUGGCCCGAGGGACCGA & CUGCCCUGGCCCGAGGGACCGA \\
miR-378a-3p & ACUGGACUUGGAGUCAGAAGGC & ACUGGACUUGGAGUCAGAAGG \\
miR-187-3p & UCGUGUCUUGUGUUGCAGCCGG & UCGUGUCUUGUGUUGCAGCCGG \\
miR-204-3p & GCUGGGAAGGCAAAGGGACGU & GCUGGGAAGGCAAAGGGACGUU \\
miR-873-3p & GGAGACUGAUGAGUUCCCGGGA & GAGACUGACAAGUUCCCGGGA \\
miR-338-3p & UCCAGCAUCAGUGAUUUUGUUG & UCCAGCAUCAGUGAUUUUGUUGA \\
miR-21-5p* & UAGCUUAUCAGACUGAUGUUGA & UAGCUUAUCAGACUGAUGUUGA \\
miR-224-5p* & UCAAGUCACUAGUGGUUCCGUUUAG & CAAGUCACUAGUGGUUCCGUUU \\
miR-142-5p* & CAUAAAGUAGAAAGCACUACU & CAUAAAGUAGAAAGCACUACU \\
miR-212-5p* & ACCUUGGCUCUAGACUGCUUACU & ACCUUGGCUCUAGACUGCUUACUG \\
\hline
\end{tabular}




\section{Figure 1}

Comparative transcriptome analysis of miRNA in human kidney tissues with or without renal functional injury.

Comparative transcriptome analysis of miRNA in human kidney tissues with or without renal functional injury. (A) Volcano plot showed differential expression of miRNA in human kidney tissues between normal and abnormal renal function group. Red dots = Up regulation; Yellow dots $=$ Down regulation. (B) Hierarchical clustered heat map showed the expression patterns of significantly (fold change $>1.5 ; \mathrm{P}<0.01$ ) and differentially expressed miRNA: Blue $=$ Down regulation; Yellow $=$ Up regulation; White $=$ No significant difference. Normal renal function group: N1, N2, N3; Abnormal renal function group: S1, S2, S3. 


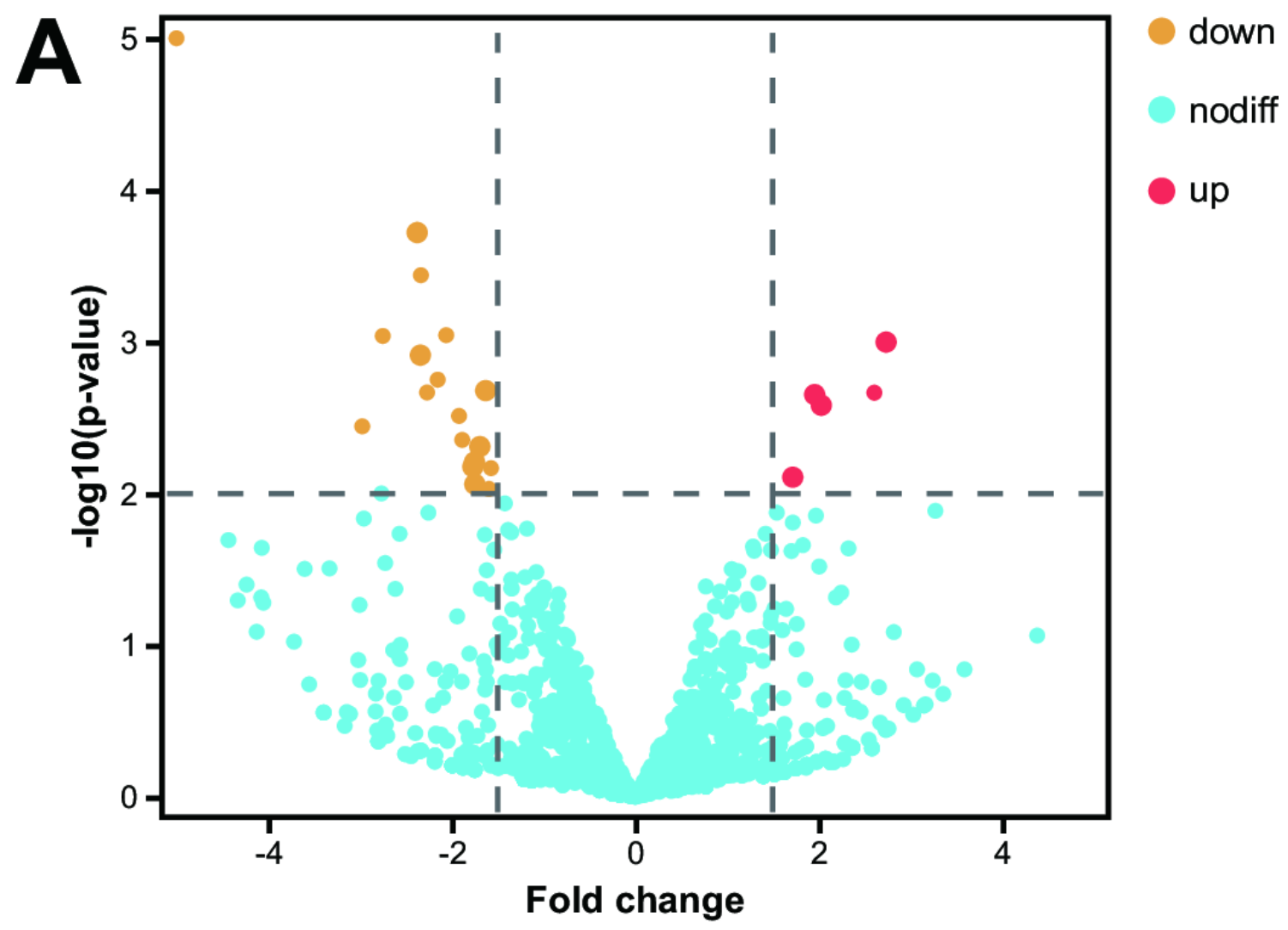

B

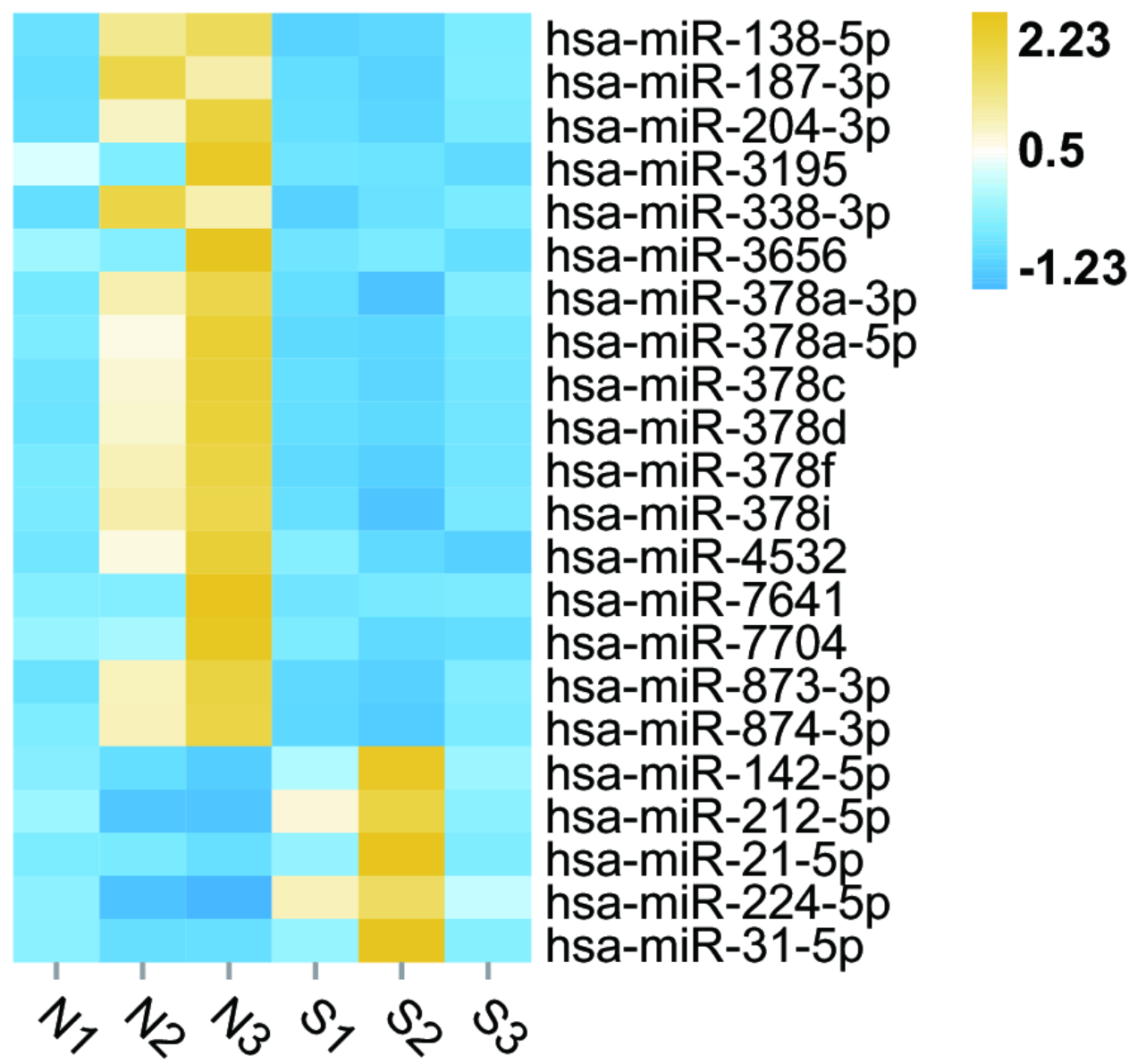


Figure 2

GO analysis and KEGG pathway analysis of the miRNA-mRNA Network.

(A) GO enrichment analysis of biological process, molecular function and cellular compartments. Blue bars represent the number of biological processes related genes;

Orange bars represent the number of molecular function related genes; Green bars represent the number of cellular component related genes; (B) Barplot of KEGG pathway enrichment. 


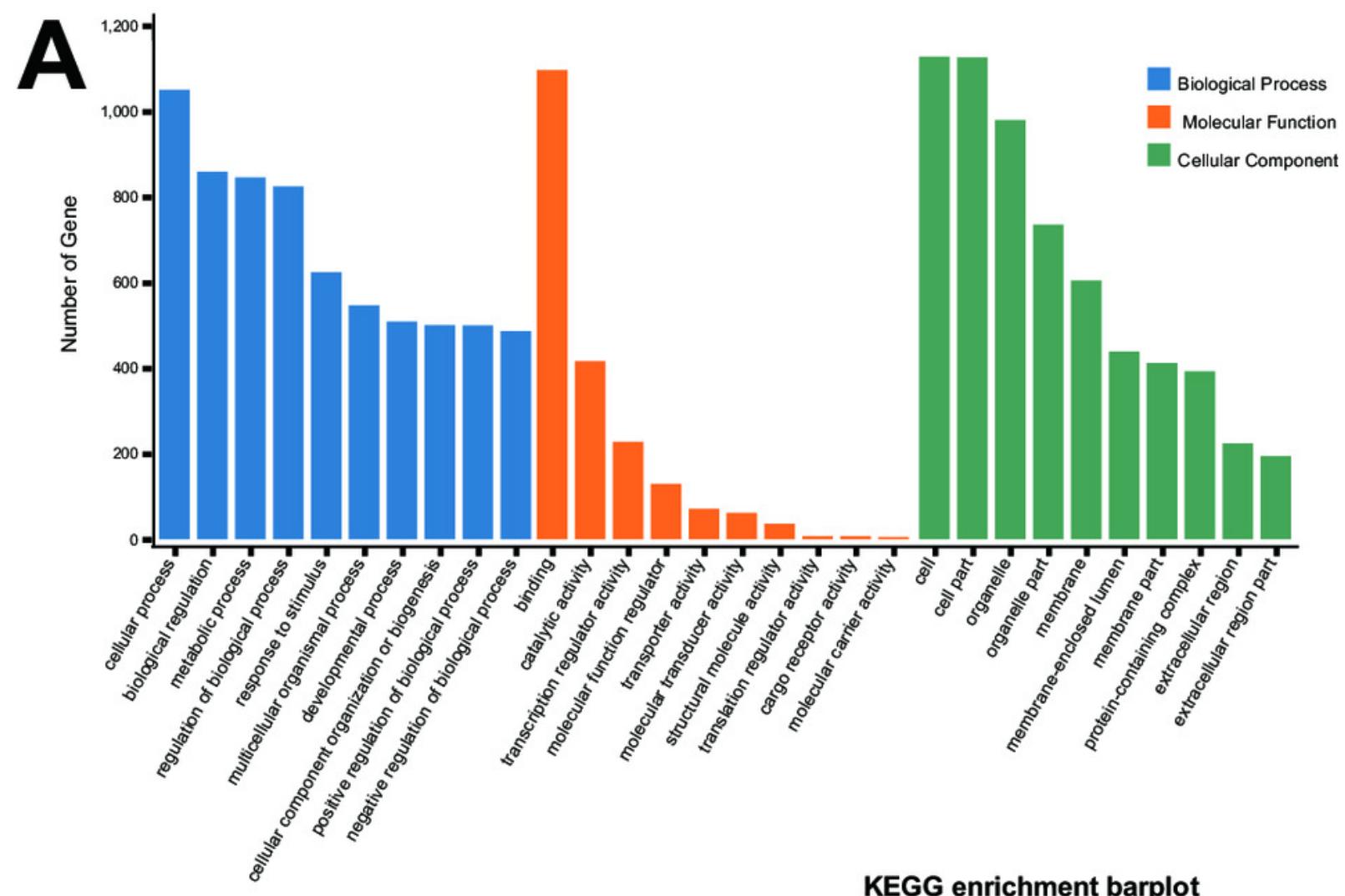

B

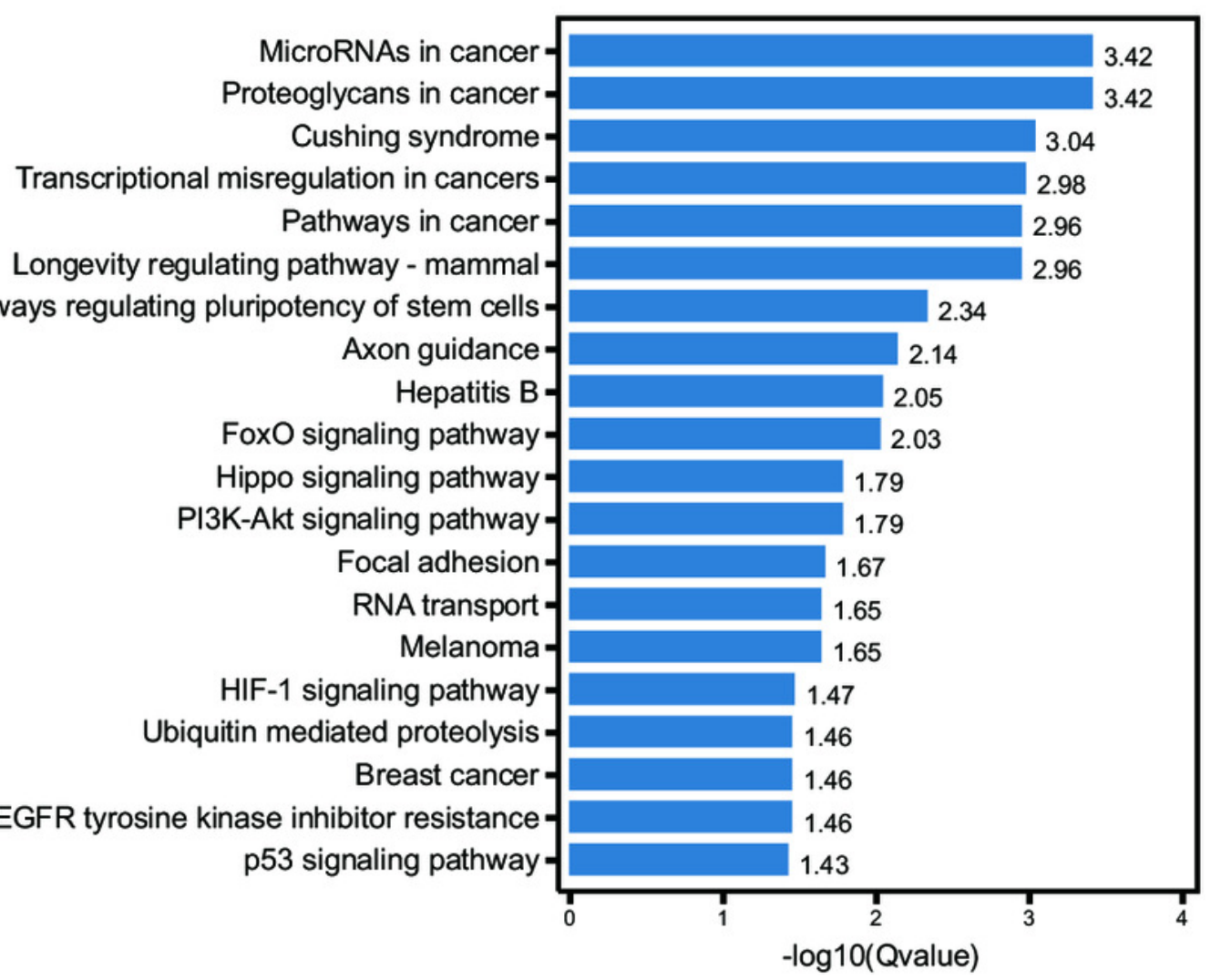


Figure 3

RT-qPCR of miRNAs in kidney tissues and HK-2 cell line.

(A) Changes of miRNA expression in kidney tissue on UUO 2d; (B) Changes of miRNA expression in kidney tissue on UUO 5d; (C) RT-qPCR of miR-21-5p, miR-142-5p, miR-187-3p in kidney tissues of patients with congenital hydronephrosis; (D) RT-qPCR of miR-187-3p in HK-2 cells induced by TGF- $\beta 1$ in vitro.

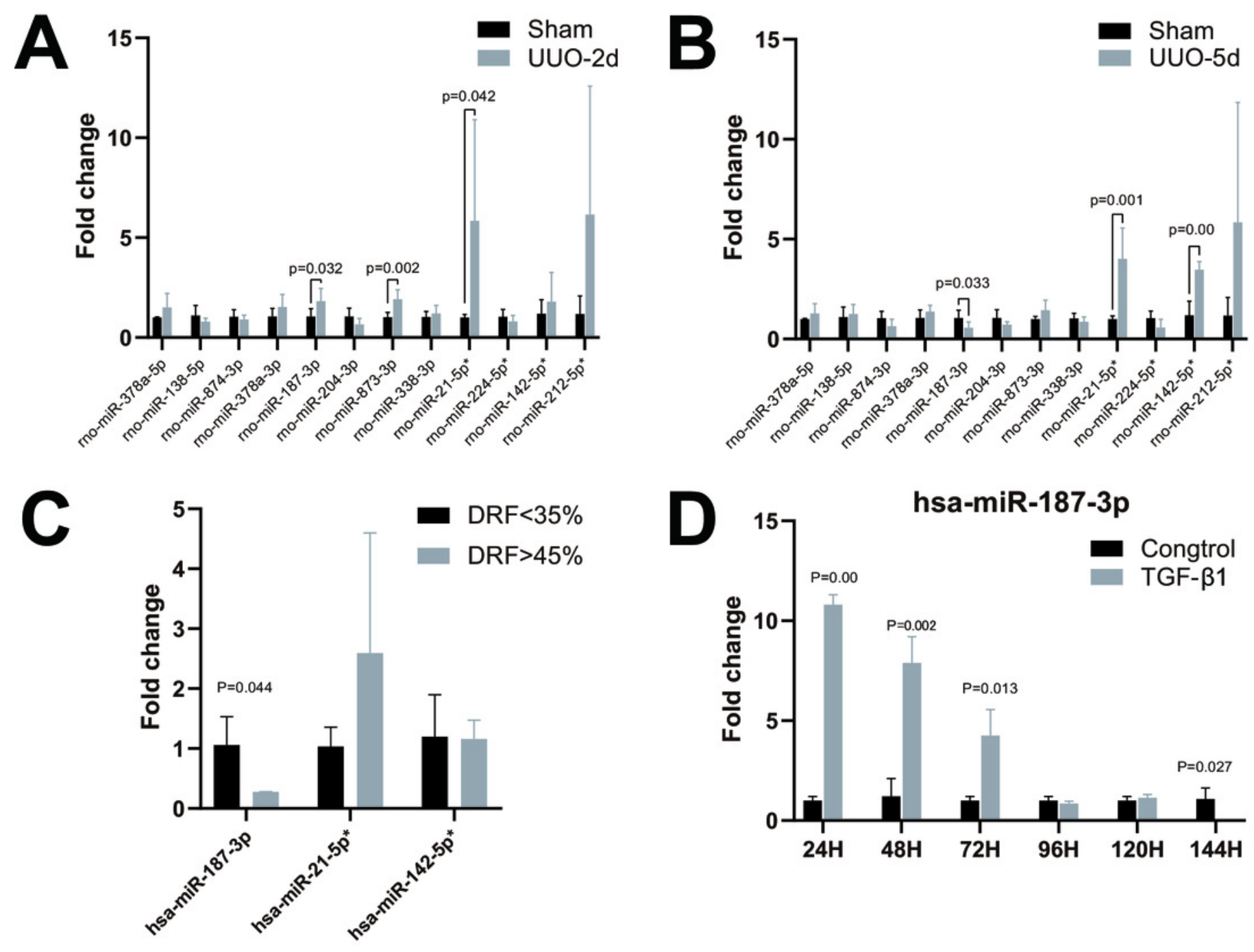

